\title{
Une perspective historique sur la polyphonie
} géorgienne

Georgian polyphony in historical perspective

\section{Susanne Ziegler}

Traducteur : Isabelle Schulte-Tenckhoff

\section{OpenEdition \\ Journals}

Édition électronique

URL : http://journals.openedition.org/ethnomusicologie/1383

ISSN : 2235-7688

Éditeur

ADEM - Ateliers d'ethnomusicologie

Édition imprimée

Date de publication : 31 octobre 1993

ISBN : 2-8257-0485-7

ISSN : 1662-372X

\section{Référence électronique}

Susanne Ziegler, "Une perspective historique sur la polyphonie géorgienne », Cahiers

d'ethnomusicologie [En ligne], 6 | 1993, mis en ligne le 02 janvier 2012, consulté le 01 mai 2019. URL: http://journals.openedition.org/ethnomusicologie/1383

Ce document a été généré automatiquement le 1 mai 2019.

Tous droits réservés 


\section{Une perspective historique sur la polyphonie géorgienne}

Georgian polyphony in historical perspective

\section{Susanne Ziegler}

Traduction : Isabelle Schulte-Tenckhoff

\section{NOTE DE L'ÉDITEUR}

Traduit de l'allemand. C'est le système anglais de translitération des mots géorgiens qui est utilisé ici.

\section{Introduction}

1 Grâce à une conception élargie de la discipline, la recherche historique dans le domaine des musiques de tradition orale a récemment gagné en importance: des études systématiques sur le sujet existent depuis le milieu des années soixante ${ }^{1}$. Mais on peut faire l'histoire de ces musiques sous divers angles d'approche, selon la région géographique considérée et l'état des sources. Dans la plupart des cas, la recherche porte sur des textes fournissant des données au sujet des musiques du passé, comme les récits de voyage et les sources judiciaires, et même les notations anciennes ${ }^{2}$. Jusqu'à présent, les documents sonores historiques n'ont pas souvent fait l'objet d'une étude, pour la simple raison que de tels matériaux n'existent que depuis la fin du siècle dernier. De nombreux enregistrements anciens demeurent difficiles d'accès et souvent en mauvais état. Les quelques travaux qui leur sont consacrés les abordent généralement sous leurs aspects acoustiques et documentaire (Schüller 1974 ; Brandl 1986; Thiel 1986); très rarement, référence y est faite aux enregistrements eux-mêmes. 
2 Dans le présent essai, on tentera une comparaison entre documents sonores anciens et actuels, fondée sur des enregistrements réalisés dans la même région de Géorgie et à propos du même répertoire. La collection historique comprend vingt-trois chansons populaires enregistrées en 1935 à Leningrad (aujourd'hui Saint-Pétersbourg); la collection récente est le fruit d'une recherche sur le terrain menée en Géorgie en été 1991 3 .

3 La polyphonie géorgienne se prête particulièrement bien à une étude historique. Depuis le milieu du siècle passé, il existe en Géorgie quantité de sources écrites sur la musique populaire, qui comprennent des collections aussi bien que des traités sur les chansons populaires. Les premiers enregistrements sur phonographe datent de 1902 ; ils ont été réalisés par deux compositeurs géorgiens, Dimitri Arakishvili et Zakhari Paliashvili Malheureusement, ces documents ne sont plus disponibles aujourd'hui, mais il existe des enregistrements réalisés en studio en 1907-1914 avec des ensembles célèbres, ayant récemment fait l'objet de rééditions (Erkomaishvili 1987). Il existe, en outre, des documents sonores anciens conservés à l'extérieur de la Géorgie, telles les célèbres collections de prisonniers de guerre russes de la Première Guerre mondiale réunies à Vienne (Lach 1928) et à Berlin (Nadel 1933) : ce sont de précieux documents historiques, dont toute comparaison diachronique doit tenir compte.

4 Mais il ne faut pas oublier qu'en raison de ses nombreux contacts avec l'Europe, la Géorgie a très tôt subi l'influence de la musique classique (l'année 1851 vit la fondation de l'Opéra de Tiflis, aujourd'hui Tbilissi), qui a eu de fortes répercussions sur la polyphonie vocale populaire. C'est pourquoi les enregistrements historiques peuvent servir à résoudre des questions litigeuses car, en dépit de leurs imperfections techniques, ils n'en fournissent pas moins des indications sur les anciennes pratiques vocales.

\section{Les enregistrements historiques}

5 En juillet 1935, trois ethnomusicologues, Ernst Emsheimer, Evgenij Gippius et Zinaida Ewald, accompagné d'un linguiste géorgien, Josif Megrelidze, enregistrèrent à Léningrad un groupe de chanteurs gouriens de Géorgie. Appartenant aux styles vocaux ouestgéorgiens, la polyphonie gourienne se signale par l'indépendance extrême des voix individuelles, ainsi que par la complexité de leurs structures mélodiques. C'est pourquoi ces chansons sont interprétées le plus souvent par un trio d'hommes exécutant chaque partie en soliste 5 .

6 La particularité des enregistrements de Leningrad tient au fait que les chanteurs furent enregistrés simultanément avec trois phonographes, si bien que chaque voix a été gravée individuellement sur un cylindre en cire. Cette technique d'enregistrement est qualifiée aujourd'hui d'analytique; je pense que les documents en question sont parmi les premiers enregistrements de ce type dans notre discipline. Ernst Emsheimer (musique) et Josif Megrelidze (texte) avaient l'intention de les publier, ce qui n'a cependant pas été fait à ce jour. La collection, comprenant une copie sur bande magnétique des enregistrements originels assortie de leurs transcriptions (mais malheureusement dépourvue de toute documentation) me fut transmise en 1987 par Ernst Emsheimer lui-même. Tous les originaux (cylindres, transcriptions et manuscrits) sont conservés aux archives sonores de Saint-Pétersbourg. Dans le cadre de mes recherches, j'ai effectué des séjours d'étude en Géorgie tout comme à Saint-Pétersbourg. A Tbilissi, je suis allée voir plusieurs fois le 
professeur Josif Megrelidze, le seul survivant de l'équipe, qui m'autorisa à consulter son manuscrit de 1936 contenant de nombreuses informations sur les chanteurs ${ }^{6}$.

Fig. 1 : Ermalo Sikharulidze, interprète de la voix principale

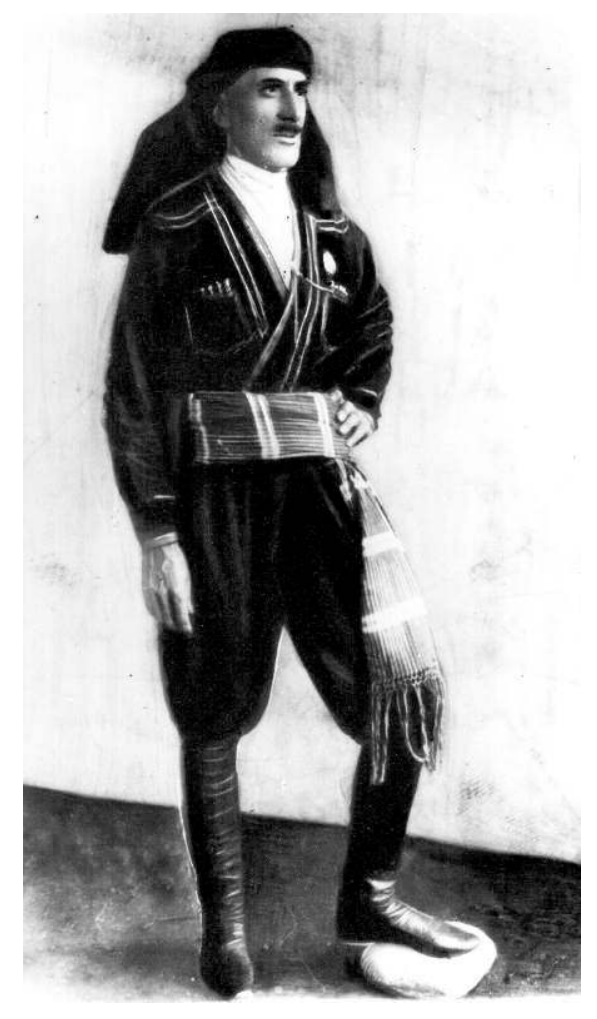

S.I.n.d. La photo provient de Géorgie

7 Constitué de cinq chanteurs, l'ensemble gourien enregistré en été 1935 à Leningrad faisait partie du célèbre Chœur populaire national de Géorgie dirigé à l'époque par Kirile Pachkoria, qui était à cette époque en tournée à Moscou et à Leningrad. Les enregistrements, réalisés à l'initiative d'Evgenij Gippius, alors directeur des archives sonores, eurent lieu les 21 et 31 juillet dans les locaux des archives. Voici les membres de l'ensemble :

1. Vladimer Lazarevich (Ladimer) Berdzenishvili, né en 1895 dans un village proche de la petite ville gourienne de Chokhatauri. Connu depuis 1919 comme chanteur professionnel, il dirigeait l'ensemble gourien. C'était une excellente basse (bani), doté d'une voix exceptionnellement étoffée. «Sa voix donnait l'impression que plusieurs basses à voix également fortes chantaient dans le chœur » (Megrelidze 1936 : 63).

2. Ermolay Ivanovich (Ermalo) Sikharulidze, né en 1883 à Chokhatauri, interprète de la deuxième voix ou voix principale (mtkmeli). Sa voix est décrite comme agréable et plutôt douce (fig. 1).

3. Feofan Ivanovich Lomtatidze (Teopile), né en 1877 près de Chokhatauri, qui avait la réputation d'être un excellent chanteur de la voix supérieure (krimantchouli). Il possédait une voix soutenue, claire et de grande portée qui s'harmonisait parfaitement avec celle de Ladimer Berdzenishvili. "Il chante sans effort et, si l'auditeur ignore que Teopile est le chanteur de krimantchouli, il pourrait avoir l'impression que celui-ci reste silencieux, alors que la voix de krimantchouli atteint son apogée. Il ouvre un tout petit peu la bouche et chante sans remuer les lèvres, avec une voix qui rappelle le son d'une flûte... On ne rencontre pas 
souvent un bon chanteur de krimantchouli, mais un chanteur comme Lomtatidze est vraiment une exception rare " (Megrelidze 1936:64; fig. 2).

4. Ushangi Simonovich Shevardnadze, né en 1886 près d'Ozurgeti (Gourie du sud) et interprète de la deuxième voix (mtkmeli). Il possède une voix légèrement vibrante et plutôt basse, mais qui s'intègre bien au son de l'ensemble.

5. Mikhail Ermolaevich Koroshinadze, né en 1907 près de Chokhatauri et également chanteur de basse (bani), le plus jeune du chœur. Sa voix s'harmonisait surtout avec celle d'Ushangi Shevardnadze.

Fig. 2 : Teopile Lomtatidze, interprète de la voix krimatchouli

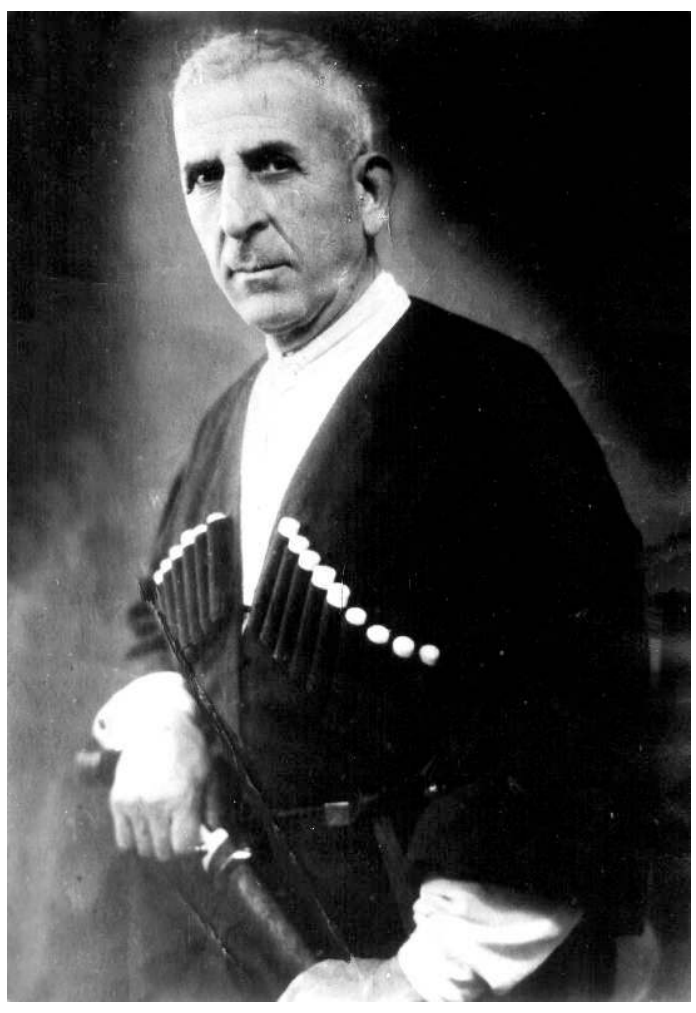

S.I.n.d. Don de la famille du chanteur

Les trois premiers chanteurs se connaissaient depuis leur jeunesse et formaient un trio depuis 1905, avant de devenir des professionnels. Pendant les enregistrements de Leningrad, c'est eux qui interprétèrent la plupart des chansons. Les deux autres furent spécialement invités pour la tournée, leur tâche consistant à assurer la partie de chœur gadatsakhili, qui n'apparaît pas dans toutes les chansons.

Composé de vingt-trois chansons populaires gouriennes à plusieurs voix, le répertoire enregistré à Léningrad présentait différents genres vocaux. Presque toutes les chansons $\mathrm{du}$ répertoire existent dans de nombreuses versions; on en trouve la trace dans des collections plus ou moins anciennes, et il en existe aussi des enregistrements. Parmi elles figurent des chansons de table comme "Mravalzhamier" (Longue vie), des chansons historiques comme "Alipasha", "Hasanbegura " et "Wakhtanguri", ainsi que des chansons saisonnières de la tradition locale, comme "Alilo", un chant de Noël, "Maqruli", un chant de mariage, ou "Agideli», une chanson accompagnant les vendanges; en raison de la situation politique prévalant à l'époque, il y avait aussi une chanson à la gloire de Staline et une à celle de Lénine. 
Fig. 3 : « Alipasha », chanson historique gourienne, enregistrée en juillet 1935 aux archives sonores de Léningrad, enregistrement $A$ (de type analytique)

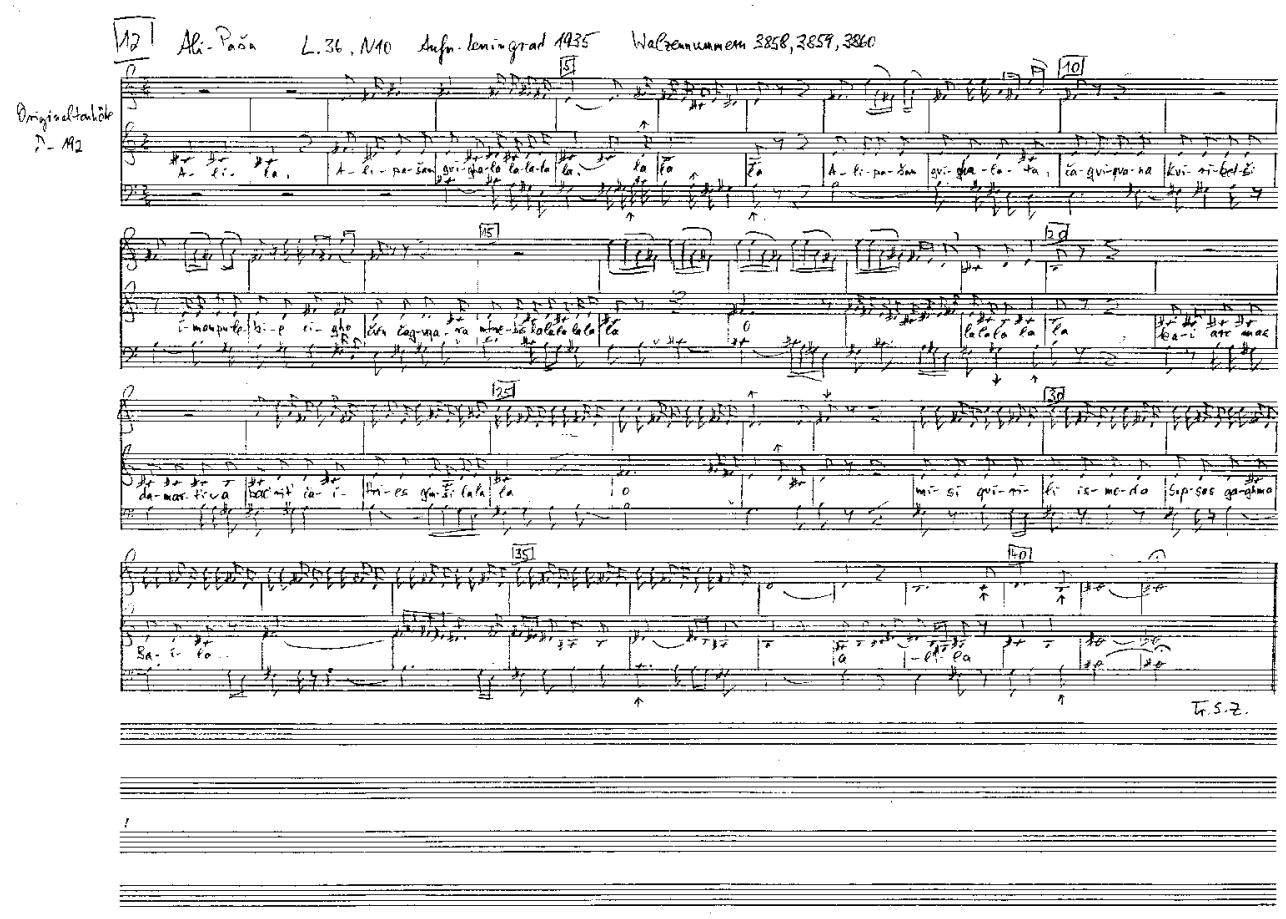

Interprètes : krimantchouli (cylindre no. 3858 d) Teopile Lomtatidze ; mtkmeli (cylindre no. 3859 c) Ermalo Sikharulidze ; bani (cylindre no. 3860 d) Ladimer Berdzenishvili.

Pour toutes les chansons enregistrées, il existe une version des trois voix enregistrées individuellement et une autre de l'ensemble au complet, réalisée par la suite. Sur les enregistrements individuels, on n'entend que rarement les autres voix, ce qui donne à penser que les phonographes étaient placés à une certaine distance l'un de l'autre. Les transcriptions effectuées sur la base des enregistrements individuels représentent ainsi des versions dont il n'existe pas d'enregistrement d'ensemble. Inversement, on ne trouve pas de transcription des versions d'ensemble, étant donné que les parties sont difficiles à distinguer et que les enregistrements de l'ensemble portent sur des variantes différentes de celles enregistrées séparément.

11 Prenons comme exemple le chant historique "Alipasha", composé à la fin du siècle passé, qui évoque des épisodes de la guerre russo-turque. En voici le texte :

\begin{tabular}{|l|l|}
\hline Alipasham gvighalata, & Ali Pasha nous a trahis, \\
chagviqvana Kvirikeshi, & il nous a emmenés à Kviriketi ; \\
imam pulebi aigho, & il a pris de l'argent \\
chven chagvqara mtrebis khelshi, & et il nous a fait tomber entre les mains de [l'ennemi. \\
kai arc mas damartia ; & Mais il ne s'en porte pas mieux, \\
bacrit chaitries gemshi. & avec une corde ils l'ont traîné dans le bateau. \\
misi qvirili ismoda & On l'a entendu hurler \\
Supsas gaghma Bailetshi & au delà de la Supsa à Baileti'. \\
\hline
\end{tabular}


La chanson est composée de trois voix indépendantes :

1. la voix krimantchouli (litt. «falsetto pendant lequel on tord le visage ») chantée par Teopile Lomtatidze. Elle est la plus aiguë du tissu polyphonique, émettant en l'occurrence des formules très rapides (sauts de septième) dans le registre aigu ; mais les notes sont faciles à distinguer, en dépit du caractère extrême du registre et de la vitesse d'exécution. La voix de krimantchouli est difficile à chanter, et de bons interprètes ont toujours été rares en Gourie. Dans l'image globale de la chanson, elle remplit une fonction ornementale; c'est pourquoi ses paroles ne forment pas un texte cohérent, mais consistent généralement en des syllabes dépourvues de signification.

2. la voix mtkmeliou damtskhebi (litt. « qui commence »), interprétée par Ermalo Sikharulidze et appelée de manière neutre «deuxième voix » dans la documentation; parfois, elle est désignée comme ténor ou voix principale. Ce n'est pas la voix la plus aiguë, bien qu'elle soit notée comme telle dans les chansons dépourvues de voix krimantchouli, mais c'est elle qui mène le chant et qui introduit le plus souvent la chanson; c'est aussi la seule voix qui donne le texte complet.

3. La voix bani (basse) interprétée par Ladimer Berdzenishvili, qui joue un rôle prédominant par son timbre pénétrant et son registre grave. Un bon chanteur de basse doté d'une riche expérience, comme le fut Berdzenishvili, est indispensable à la bonne exécution d'une chanson gourienne. Dans le chant populaire gourien, à l'opposé de la polyphonie classique européenne, ce n'est pas seulement la voix de basse qui détermine l'harmonie; celle-ci est plutôt produite par l'ensemble des voix, donnant lieu à des accords spéciaux parfois surprenants (cf. fig. 3) $)^{8}$.

Les trois voix furent transcrites individuellement et notées sur portées. Étant donné que les autres voix ne peuvent être entendues que rarement et ne peuvent donc servir de référence, la transcription des voix individuelles est rendue difficile : les gammes ne sont pas tempérées, la hauteur du son est élastique; il en résulte souvent des tournures harmoniques inattendues. Les seuls points de référence sont les fins de phrase où les trois voix sonnent en général à l'unisson ou en intervalle de quinte.

Il n'existe pas de transcription de l'enregistrement complet, qui diffère d'ailleurs à maints égards de la version considérée ici. En l'occurrence, il y a des différences considérables entre les deux versions, chantées l'une après l'autre; ce qui confirme l'idée de la pérennité, à l'époque, de l'improvisation spontanée par les chanteurs, même dans une chanson à plusieurs voix du répertoire standard.

Les enregistrements en question représentent toujours un point fort de la polyphonie gourienne, en raison de la maitrise virtuose du répertoire, des qualités vocales et de la riche expérience dont les interprètes font preuve dans le chant d'ensemble, et aussi en raison des conditions particulières de l'enregistrement.

\section{Les enregistrements actuels}

Grâce à l'appui financier de la Deutsche Forschungsgemeinschaft, j'ai pu effectuer en juin 1991 un séjour de terrain en Gourie, en collaboration avec le département de folklore du Conservatoire national de Tbilissi ${ }^{9}$. Notre destination fut la petite ville de Chokhatauri en Gourie du nord, d'où étaient originaires la plupart des interprètes des enregistrements de 1935. Nous avions l'espoir d'y trouver le plus grand nombre possible de variantes des chants enregistrés à Leningrand, et de recueillir davantage de renseignements sur les chanteurs, ainsi que sur les répertoires d'alors et d'aujourd'hui. Pour obtenir les meilleurs 
enregistrements possibles, nous avons utilisé simultanément trois appareils, un AIWADAT Recorder pour l'enregistrement de l'ensemble au complet et deux magnétophones à cassettes Sony WM-D6C munis chacun d'un mini-microphone par canal à attacher à la chemise du chanteur, pour l'enregistrement des voix individuelles. L'étude des enregistrements anciens nous avait appris qu'il est malaisé de transcrire une voix indépendamment des points de repère fournis par les autres voix. C'est pourquoi nous n'avons pas complètement séparé les voix mais enregistré chacune d'entre elles sur un canal, si bien qu'on peut aussi en entendre deux en stéréo ${ }^{10}$.

Le résultat de la mission fut tout à fait positif : à Chokhatauri et dans les environs, nous avons pu enregistrer 78 chansons gouriennes interprétées par des chœurs (professionnels ou non) aussi bien que par des chanteurs individuels formant des trios. Nous avons réussi, entre autres, à enregistrer une quarantaine de variantes des quinze chansons de la collection d'Emsheimer; certaines d'entre elles ne furent chantées qu'une fois, d'autres cinq ou même six fois. Il est apparu que seules deux chansons ("Agideli» et "Chokhataura ») étaient tout à fait inconnues dans le répertoire actuel ; les chansons sur Lénine et Staline étaient connues, mais pour des raisons évidentes, on ne tenait plus à les interpréter. Trois autres chansons ne purent pas être enregistrées, car elles exigeaient de meilleurs interprètes de krimantchouli qui, malades, n'étaient pas disponibles lors de notre passage.

Parmi les chansons dont nous avons pu recueillir plusieurs versions, il y a "Alipasha", enregistrée en trois formations : 1 . le Chœur des vétérans du travail et de la guerre, à Chokhatauri, composé exclusivement d'hommes âgés ; 2 . les membres de l'ensemble vocal le plus largement connu localement, l'ensemble Guria, qui a déjà réalisé un disque ; 3. trois hommes âgés entre 70 et 90 ans, originaires du village de Tsipnari près de Chokhatauri, qu'on fit venir spécialement - l'un d'eux fut même arraché à son lit de malade - pour chanter pour nous. D'une manière générale, la comparaison des ensembles et des répertoires a révélé que les chanteurs plus âgés (ici: formations 1 et 3) connaissaient des versions plus anciennes des chansons, voire même les versions chantées en 1935, bien que d'une façon moins parfaite. Pour une comparaison avec les enregistrements anciens, j'ai choisi la version no. 2 (cf. fig. 4), car les membres de cet ensemble étaient les seuls à atteindre, du point de vue de leurs qualités vocales, de leur assurance et de leur maîtrise du répertoire, un niveau comparable à celui des enregistrements anciens. Cette version d'«Alipasha » fut enregistrée le 21 juin 1991 à la maison de la culture de Chokhatauri, avec un trio de l'ensemble Guria, dont les membres se considéraient d'ailleurs tous comme solistes. La voix principale (tskheba) était tenue par Wazha Gogoladze, le directeur de l'ensemble, la voix de krimantchouli par Ivane Gudavadze, et la voix de basse bani par Tariel Kilasonia. Selon la pratique actuellement en vigueur, "Alipasha» figure dans notre enregistrement avec une autre chanson gourienne, « Adila ». 
Fig. 4 : « Alipasha », chanson historique gourienne, enregistrée le 21 juin 1991 à la maison de la culture de Chokhatauri (Gourie) par S. Ziegler (juin 1991 IV.10, enregistrement à la fois analytique et global).

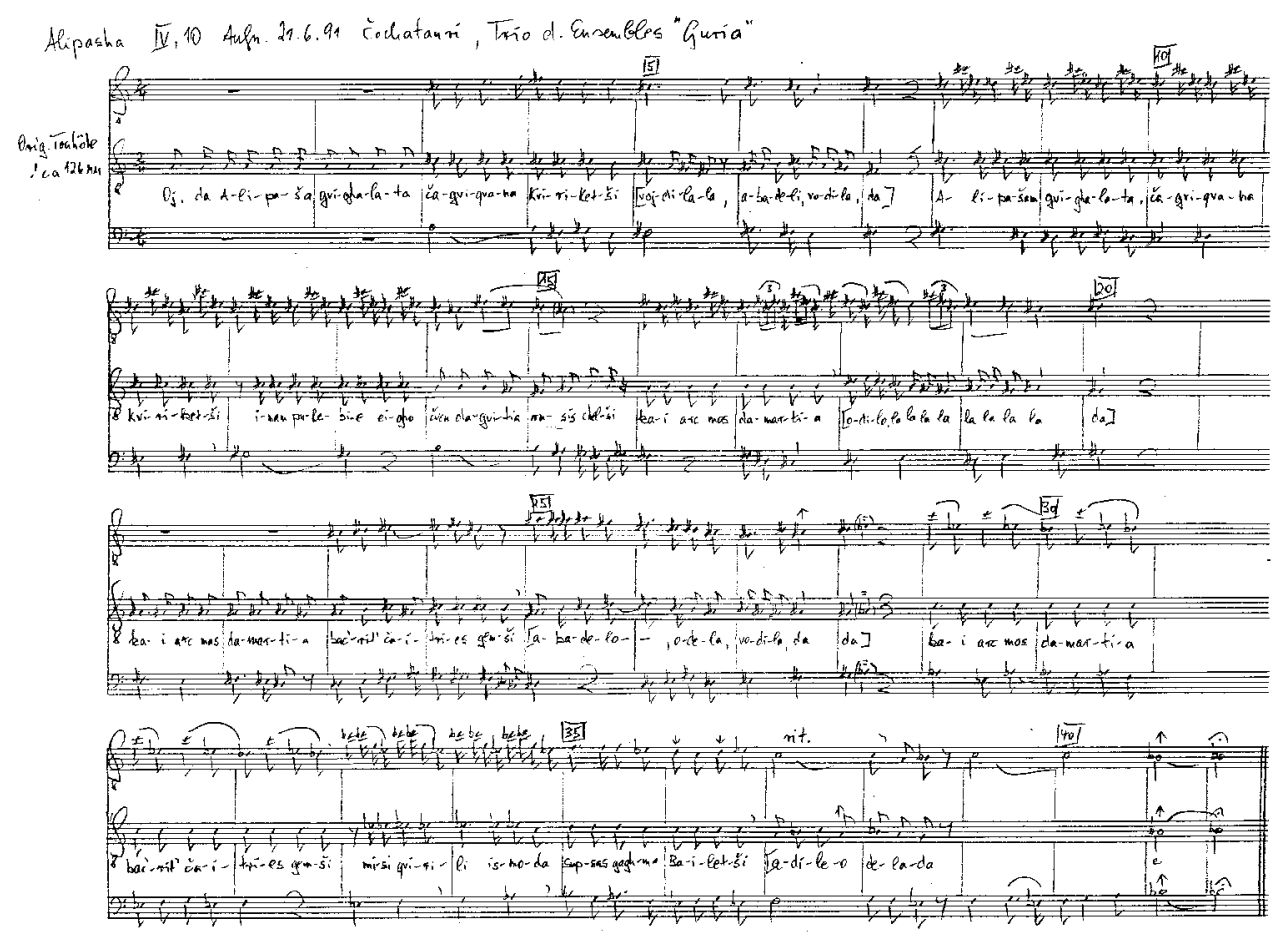

Interprètes: krimantchouli, Ivane Gudavadze; tskheba, Wazha Gogoladze; bani, Tariel Kilasonia.

\section{La comparaison}

Les matériaux disponibles autorisent une comparaison portant sur toutes sortes d'aspects. Indépendamment de la qualité technique, les différences entre les enregistrements de 1935 et notamment ceux de 1991 concernent, non seulement les interprètes, mais encore le mode d'exécution de la chanson du point de vue de la conduite de la voix, des échelles, de l'harmonie et des formules utilisées. Sans anticiper sur un ouvrage en cours de rédaction, je me bornerai ici à faire ressortir quelques points saillants, en prenant comme exemple la chanson « Alipasha ».

Dans la version ancienne de cette chanson, les voix évoluent d'une manière plus complexe que dans la nouvelle. Par exemple, le chanteur de la voix de krimantchouli enregistré en 1935 effectuait les sauts de septième plus fréquemment et avec plus de précision tonale et rythmique que le chanteur enregistré en 1991, qui n'était pas sûr de lui et n'exécutait qu'occasionnellement des sauts de septième à la manière traditionnelle (mesures 8-13, 33-34). Dans la version ancienne, le chanteur de la voix principale ( damtskhebi) ne se borne pas à réciter le texte à une hauteur du son déterminée, mais il développe aussi des formules mélodiques (mesures 12-16, 33-37) qui rendent le texte secondaire. La cohérence des voix devient ainsi plus difficile à assurer, car pareille liberté vocale n'est possible que si chaque chanteur s'est bien familiarisé avec la façon dont ses collègues structurent leur partie et que tout l'ensemble a acquis une certaine routine dans le chant collectif. Quant à la voix de basse bani, on constate une évolution rythmique et harmonique progressive vers une fonction d'appui: dans la version ancienne 
prédominent des sections mélodiques avec des notes d'une durée plus brève, tandis que dans la version plus récente, les notes sont d'une durée plus longue, accentuant l'univocité des harmonies (mesures 10-15, 29-37). Cela résulte en partie aussi du tempo plus rapide de l'enregistrement récent. Une telle accélération des tempos, ainsi que l'élévation de la hauteur du son absolue, loin d'être des cas isolés, représentent actuellement une tendance générale.

Une comparaison des voix individuelles montre que l'indépendance de chaque voix était jadis nettement plus marquée. La qualité professionnelle et la grande expérience dans le chant d'ensemble que possédaient les interprètes de 1935 étaient une base idéale pour l'improvisation individuelle dans les limites de la version établie. Elle se manifeste par une plus grande liberté dans l'ornementation de la mélodie, par des tournures inattendues dans la construction des harmonies et une plus grande liberté de modulation, par des échelles hautement variables et difficiles à fixer par écrit (il ne s'agit pas de la gamme tempérée largement connue aujourd'hui), et par le fait qu'une chanson n'est jamais répétée mais émerge en des variantes toujours renouvelées. Les versions actuelles, en revanche, surtout celles interprétées par des ensembles folkloriques nationaux, représentent le plus souvent des versions standardisées laissant peu de place à l'improvisation individuelle. De ce fait, elles ne sont donc plus caractéristiques d'une région, voire d'un chanteur spécifique, mais sont interprétées d'une manière uniforme à travers tout le pays. Ainsi, les versions des chansons présentées par l'ensemble Guria diffèrent souvent considérablement de celles que nous avons enregistrées à Chokhatauri avec des chanteurs moins professionnels et souvent plus âgés. Il semblerait que les versions autochtones ne soient que très rarement devenues des modèles dont s'inspirent les ensembles locaux ; on a plutôt tendance aujourd'hui à se baser sur les versions de plus en plus standardisées des chansons du répertoire des ensembles folkloriques nationaux.

Les enregistrements de 1935 furent réalisés avec les chanteurs gouriens les plus célèbres de leur temps, tous des professionnels. Aujourd'hui, de tels interprètes sont rares. On trouve encore de bons chanteurs, mais la plupart d'entre eux font partie d'ensembles, si bien qu'ils ont peu de possibilités d'élaborer leurs propres variantes. En raison d'une mobilité accrue, on ne rencontre plus guère aujourd'hui des chanteurs qui forment depuis longtemps une communauté de chant (parents, amis de jeunesse, collègues de travail), comme c'était le cas jadis. Nos recherches montrent que de telles communautés n'ont survécu que parmi les hommes âgés qui, ayant perdu leurs qualités vocales, ne peuvent ainsi plus être choisis pour des enregistrements. Les séances d'enregistrement organisées avec des chanteurs maîtrisant bien le répertoire mais ne se constituant en ensemble que pour la circonstance ne donnent jamais de résultats aussi satisfaisants que celles réalisées avec des interprètes chantant ensemble depuis des années et unis par d'autres liens que ceux de la pratique vocale commune ${ }^{11}$.

Pour placer l'analyse des deux versions d'«Alipasha" dans un contexte musicohistorique plus large et compléter ainsi la comparaison, voyons brièvement quelquesunes des nombreuses variantes de cette chanson nous paraissant significatives. 
Fig. 5 : Ensemble gourien dirigé par Artem Erkomaishvili. Géorgie, 1927

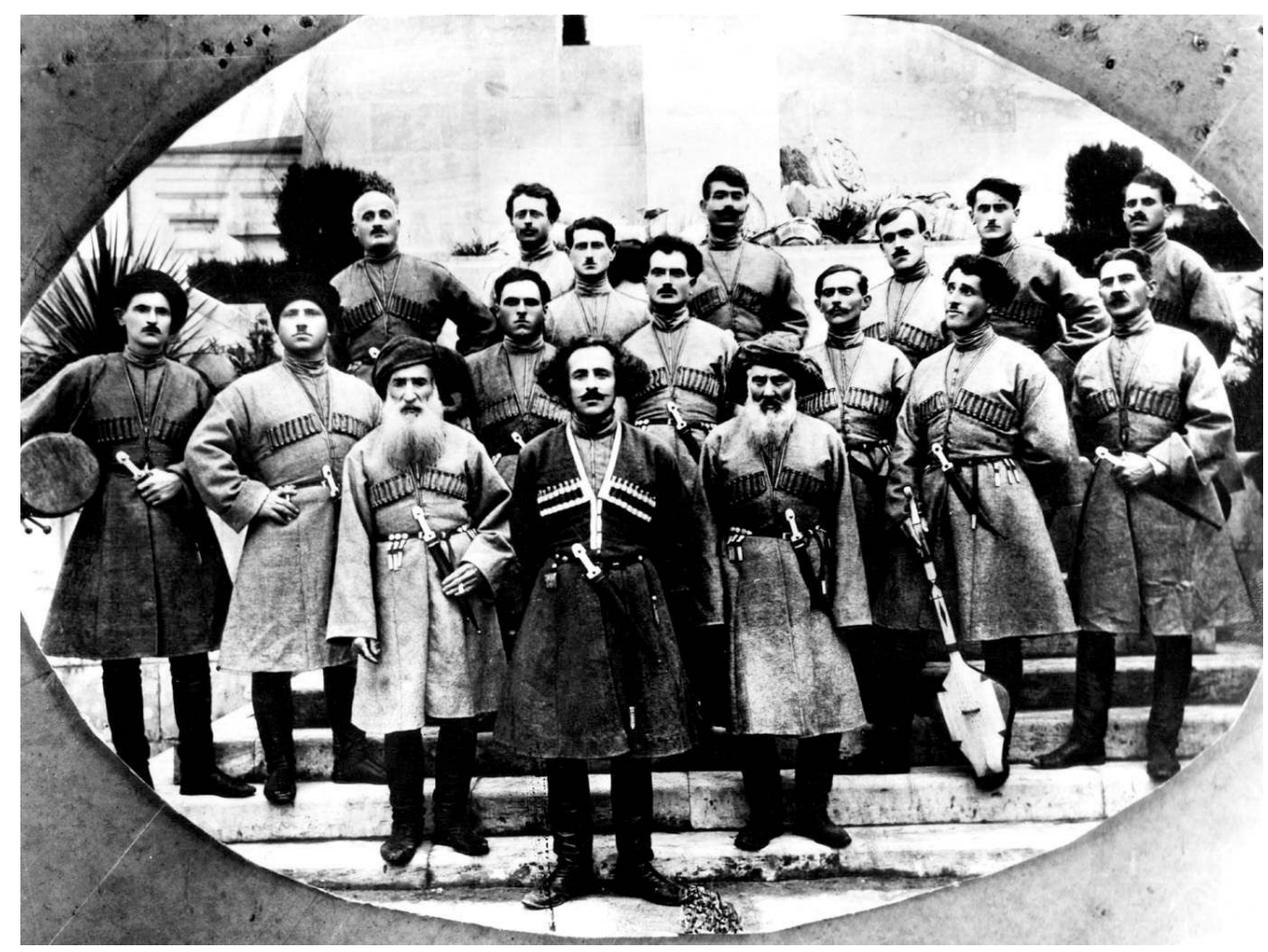

Les notations les plus anciennes que j'aie pu trouver proviennent d'une des collections de 1910 du compositeur et musicologue géorgien Zakhari Paliashvili (Paliashvili 1910 : 24ss). Ici, la ligne mélodique des voix est assez simple en comparaison avec les deux versions mentionnées plus haut; en particulier la voix la plus aiguë ne présente aucun caractère $\mathrm{du}$ type krimantchouli. D'autre part, la forme rythmique et harmonique de cette transcription donne une image nettement simplifiée. Impossible de savoir si, dans le cas présent, c'est la notation qui a été simplifiée, ou si la chanson a été interprétée dans une variante tout différente, étant donné que les documents sonores correspondants ont disparu.

Une version incomplète se trouve aussi chez Lach (1928: 93) qui n'a transcrit que la voix principale (damtskhebi), c'est-à-dire celle qui fournit le texte complet. Comme dans d'autres versions, les formules mélodiques assorties au texte en continu sont plutôt conçues sous forme de récitatif, tandis que celles accompagnant des syllabes dépourvues de signification montrent plus de mouvement. Cette version ne figurant pas parmi les chansons sélectionnées pour l'enregistrement, nous ne pouvons pas en dire davantage.

Une version fort intéressante fut publiée par Siegfried Nadel (1933 : 18ss) sur la base des enregistrements que la Berliner Phonogrammkommission entreprit, en 1918, avec des prisonniers de guerre russes en Allemagne. Au département d'ethnomusicologie du Musée d'ethnographie de Berlin (l'ancien Phonogrammarchiv de Berlin), une copie de cet enregistrement a été trouvée sous forme de bande magnétique (cote actuelle: WU 0183 II). Une comparaison du document sonore avec la transcription établie par Nadel montre toutefois combien il est difficile de transcrire cette chanson, ne serait-ce que du point de vue de la partition : un étranger à cette musique - comme Nadel - n'est pas en mesure de savoir quelle voix chante quelle note et quelle est l'évolution mélodique de chaque voix, si bien que la transcription de Nadel diffère considérablement de toutes les autres. 
variante géorgienne récente est la transcription réalisée par Anzor Erkomaishvil (1980 : 50ss). Ce natif de la Gourie du sud a relancé avec son ensemble Rustavi la tradition vocale séculaire de sa famille. La version notée par lui ne diffère que légèrement de celle que nous avons enregistrée avec l'ensemble Guria. Grâce à des tournées, à des émissions de radio et à des disques, les variantes de l'ensemble Rustavi sont connues à travers toute la Géorgie et sont devenues des modèles auxquels se réfèrent de nombreux ensembles locaux.

Pour ce qui est des documents sonores, hormis ceux réalisés par la Berliner Phonogramm après la Première Guerre mondiale, un disque de 1907 réédité en 1987 mérite d'être signalé (cf. Erkomaishvili 1987, pl. IV, n³). Ici, la chanson « Alipasha » est interprétée par un trio de Gourie du sud, célèbre à l'époque, constitué par Joseb Khukhunaishvili (voix principale), Almaskhan Kukhunaishvili (krimantchouli) et Besarion Khukhunaishvili (bani), tous de la même famille et considérés comme les meilleurs chanteurs gouriens de l'époque. Les deux versions figurant sur le disque - d'ailleurs très bien restituées représentent des variantes autonomes de la chanson; seule la seconde rappelle la version que j'ai enregistrée en 1991 avec l'ensemble Guria (fig. 5).

"Alipasha» apparaît également sur de nombreux nouveaux disques $33 \mathrm{t}$. et CD présentant des chansons populaires géorgiens. Dans la plupart des cas, elle est assortie de la chanson « Adila ", qui figure également dans la collection d'Emsheimer, mais à l'époque comme chanson autonome. Dans ses versions actuelles, cette chanson combinée « AdilaAlipasha» témoigne d'une nette tendance à l'uniformisation, perceptible dans les transcriptions aussi bien que dans les documents sonores.

Notre comparaison de documents écrits et sonores relatifs à la même chanson sur une durée de 85 ans montre clairement qu'aucune des versions n'est semblable, mais que chacune d'entre elles comporte des traits distinctifs. Ainsi, chaque chanson ne relève pas seulement d'une tradition locale spécifique (dont il reste encore à déterminer l'étendue exacte pour les chansons gouriennes), mais elle est encore façonnée par la personnalité des chanteurs.

\section{Conclusion}

31 L'analyse comparative d'enregistrements anciens et récents est pertinente pour l'étude d'une forme de polyphonie complexe comme celle de Gourie, car l'état actuel de la pratique vocale se précise si on la considère par rapport à ses développements historiques. Le principal avantage de notre étude, à savoir l'existence d'enregistrements anciens et récents aussi bien que d'enregistrements analytiques, permet des recherches spécifiques que d'autres matériaux sonores historiques n'autorisent pas nécessairement. Par conséquent, ses résultats ne doivent pas être extrapolés sans autre à d'autres domaines de recherche. La découverte de nouvelles possibilités techniques de restauration des documents sonores anciens, pourtant encore controversées actuellement, ouvre de nouvelles voies à l'ethnomusicologie au delà du cadre géographique étroit de la polyphonie géorgienne, susceptibles d'encourager de manière décisive la comparaison musicologique dans une perspective historique. 


\section{BIBLIOGRAPHIE}

BRANDL Rudolph M., 1986, « Ethnohistorie des Schalls - Fragen zu einem besonderen Quellentypus ». Musicologica Austriaca $6: 173-96$.

CHKHIKVADZE Grigol, 1980, « Georgia - Folk Music ». The New Grove Dictionary of Music and Musicians 19: 361-68.

ERKOMAISHVILI Anzor, 1980, Kartuli khalkhuri simgherebi (guruli). [Chansons populaires géorgiennes de la Gourie]. Tbilissi : Khelovneba.

ERKOMAISHVILI Anzor, 1987, Pirveli grampirpitebi sakartveloshi [Les premiers disques géorgiens] 1907-1914. Plaquette accompagnant les disques Melodija M30 46903009 - M30 46911000. Tbilissi : Khelovneba.

KADEN Christian, 1990, «Die "Geburt” des neuzeitlichen Musikbegriffs nach dem Zeugnis alltagssprachlicher Quellen ». Beiträge zur Musikwissenschaft 32(4) : 262-73.

JORDANIA Iossif, 1984, « Le chant populaire géorgien, origines, évolution et tendances actuelles ». Revue internationale des sciences sociales XXXVI(3) : 569-81.

JORDANIA Iossif, 1989, Gruzinskoe traditsionnoe mnogogolosie v mezhdunarodnom kontekste mnogogolosnykh kultur ( $\mathrm{K}$ voprosu genezisa mnogogolosiya) [La polyphonie géorgienne traditionnelle dans le contexte international des cultures polyphoniques (A propos de l'origine de la polyphonie)]. Tbilissi : Izdatelstvo Tbilisskogo universiteta (en russe).

LACH Robert, 1928, Gesänge russischer Kriegsgefangener. III. Band: Kaukasusvölker. 1. Abt. « Georgische Gesänge ». Transkription und Übersetzung der georgischen Texte von A. Dirr. Wien (55. Mitteilungen der Phonogramm-Archivs-Kommission).

MEGRELIDZE Josif, 1936, Guriiskie pesni [Chansons gouriennes]. I : Issledovanie i teksty [Études et textes]. Texte dactylographié inédit.

NADEL Siegfried F., 1933, Georgische Gesänge. Lautabteilung. Leipzig : Kommissionsverlag Otto Harrassowitz.

PALIASHVILI Zakhari, 1910, Kartuli khalkuri simgherebi [Chansons populaires géorgiennes]. Tbilissi.

SCHÜLLER Dietrich, 1974, « Die Schallaufnahme als Quelle für die Ethnohistorie ». Wiener Ethnohistorische Blätter, Beiheft 3: 33-38.

SIMON Artur, 1986, «Some possibilities and limitations of historical studies in music cultures with oral/aural traditions ». In: The Oral and the Literate in Music (Y. Tokumaru \& O. Yamaguti Eds). Tokyo: Academia Music Ltd., p. 440-52.

SUPPAN Wolfgang, 1991, «Publications and activities of the ICTM Study Group on historical sources of folk music ». Yearbook for Traditional Music 23: 189-94.

THIEL Helga, 1986, « Sound documents of the Phonogrammarchiv of the Austrian Academy of Sciences as a means of instruction within informal musical education ». Ethnomusicology 30(3): 387-93. 
ZIEGLER Susanne, 1990, « The Discovery of Georgian Polyphony. Varying Trends in a Century of Research ». In: Prepublication of the VIIth European Seminar in Ethnomusicology (Berlin, October 1990), p. 509-16.

\section{NOTES}

1. Pour un aperçu des recherches historiques, menée pour la plupart dans le cadre du groupe d'étude de l'International Council for Traditional Music (ICTM) sur les sources historiques de la musique populaire, voir Suppan (1991). Pour une étude plus globale, voir Simon (1986).

2. De nombreuses indications bibliographiques concernant les types de matériaux écrits sont fournies par Suppan (1991). Voir aussi Kaden (1990) pour la place de la musique dans les récits de voyage.

3. Dans le cadre d'un projet de recherche de deux ans, financé par la Deutsche Forschungsgemeinschaft (DFG), j'ai pu étudier en détail la collection de 1935 et séjourner à plusieurs reprises en Géorgie (1991) et à Saint-Pétersbourg (1992). Le présent article résume un ouvrage en cours de rédaction, qui sera publié comme rapport du projet. Je tiens à exprimer ici ma vive reconnaissance à tous ceux qui ont contribué à la réalisation du projet: MM. les professeurs J. Kuckertz, Max Peter Baumann et Artur Simon, de Berlin, M. le professeur Rudolf Brandl, de Göttingen, M. le professeur I. Zemtsovsky, de Saint-Pétersbourg, ainsi que Mme Rusudan Tsurtsumia et M. Edisher Garakanidze, de Tbilissi.

4. Pour l'historiographie des recherches sur la musique géorgienne, $c f$. Chkhikvadze (1980) et Ziegler (1990).

5. Cf. aussi l'article de Jordania (1984) et, pour de plus amples renseignements, les travaux d'auteurs géorgiens.

6. Le texte de Josif Megrelidze est intitulé «Chansons gouriennes"; après une brève introduction sur la Gourie, il traite exclusivement des textes des chansons, en particulier des refrains (c'est-à-dire des syllabes dépourvues de signification). Une copie du manuscrit est conservée à Saint-Pétersbourg. Je tiens à remercier le professeur Megrelidze, ainsi que les collaborateurs des archives sonores de St. Petersbourg, de leurs précieux concours.

7. La traduction est adaptée à celle [en allemand] d'Adolf Dirr (voir Lach 1928: 211). Là, le quatrième vers dit: chven chagvtia rusis khelshi, «il nous a fait tomber entre les mains des Russes ", comme on le chante d'ailleurs à nouveau de nos jours.

8. La terminologie populaire utilisée pour nommer les diverses voix de la polyphonie géorgienne est très riche et n'a pas encore été étudiée complètement. Je me base ici sur des informations et des explications qui me furent communiquées de vive voix par Edisher Garakanidze. Pour l'harmonie des chansons populaires géorgiennes, voir Jordania (1987 : 47ss).

9. Je tiens à remercier vivement tous les membres de l'équipe, les hôtes géorgiens et tout spécialement Edisher Garakanidze qui prépara et organisa la mission.

10. Je tiens à remercier Simha Arom (Paris) pour ses nombreux conseils pratiques en vue de la préparation de notre mission.

11. Des entretiens avec des chanteurs à Chokhatauri ont révélé que beaucoup d'entre eux formèrent jadis leur propre trio dont les membres se sont dispersés depuis. Ainsi nous raconta-ton à propos de deux trios que nous avons enregistrés, que notre mission leur avait donné pour la première fois depuis deux ans l'occasion de chanter ensemble. 


\section{RÉSUMÉS}

Vocal polyphony in Guria, in western Georgia, is among the most fascinating polyphonic forms in the world, still little known in Western Europe. Contrary to other polyphonic traditions, the Georgian one has been documented in the (mostly local) literature since the end of last century, and sound documents exist since the beginning of this century. The author undertakes a comparison of historical and recent recordings of Gurian folk songs. The historical recordings, which date back to 1935 , were made by three ethnomusicologists using simultaneously three phonographs. The recent collection was established in 1991 in Guria, with the purpose of repeating the recordings of 1935 . In both cases, a specific recording technique has allowed to reproduce each voice exactly. With the example of one particular song, the author confronts similarities and differences between the historical and the modern versions. On a larger scale, it thus becomes possible to gain better insight into the evolution of Gurian polyphony in the course of this century.

\section{AUTEURS}

\section{SUSANNE ZIEGLER}

Susanne Ziegler a étudié la musicologie et les langues slaves à Cologne, tout en se spécialisant dans l'étude des musiques populaires d'Europe de l'est et du sud-est. Depuis 1980, elle a enseigné l'ethnomusicologie dans diverses universités allemandes. De 1983 à 1988, elle a travaillé à l'Institut de musicologie comparée de l'Université Libre de Berlin, puis elle a été professeur invité à l'Université de Heidelberg. Depuis 1990, elle étudie la polyphonie vocale géorgienne dans le cadre d'un projet de recherche de la Deutsche Forschungsgemeinschaft. Ses publications portent sur les musiques populaires des Balkans, de la Turquie et de l'ancienne Union soviétique. 